\title{
Korreferate
}

\author{
Hartwig Brandt
}

\section{Die württembergische Sozialdemokratie im Parteiensystem des frühen Kaiserreichs Eine Skizze}

Autoren, die über württembergische Zustände handeln, sprechen nicht ungern jenen ergreifenden Refrain, daß in Schwaben alles anders sei. Die württembergische Sozialdemokratie zwischen Reichsgründung und Jahrhundertwende bietet für solche Spruchweisheit ein brauchbares Exempel ${ }^{1}$.

Die Vorgeschichte der württembergischen SPD kennt nicht den Widerstreit von ADAV und Arbeitervereinen, die Spannung von Lassallescher Agitation und bürgerlichem Reformbemühen. Sie kennt auch nicht die Entfremdung von bürgerlicher und proletarischer Demokratie oder sie hat diese doch nicht zu programmatischer Schārfe herausgebildet. Die Arbeiterbewegung war vielmehr ein Stück jener politischen Kultur, wie diese vom Bürgertum dargestellt wurde. Sie war politisch der liberalen Opposition verbunden, sie war deren Wahlklientel und Bundesgenosse zugleich. Bestrebungen, diese Bindungen zu lösen, sind nur im Kleinen zu erkennen. Bis 1870 haben sie das System politischer Symbiose nicht zu verändem vermocht.

Die württembergischen Verhältnisse der 1860er Jahre lassen sich nicht unter jene Tendenz der Epoche fassen, die man als politisch-moralischen Einbruch der Partei des „Fortschritts“ definiert. Bismarcks Strategie wirkte hier auf Liberale und Demokraten eher kontraproduktiv, und auch später noch, als das Land in das Krāftefeld des Nationalstaats geriet und schließlich in diesen inkorporiert wurde, hat es erst nach und nach

\footnotetext{
${ }^{1}$ Eine Gesamtdarstellung des Gegenstandes fehlt, aber es gibt drei Arbeiten, die durch ihre zeitliche Abfolge das Fehlen nur als geringen Mangel erscheinen lassen: Wolfgang Schmierer, Von der Arbeiterbildung zur Arbeiterpolitik. Die Anfānge der Arbeiterbewegung in Württemberg 1862/63-1878 (Hannover 1970), im folgenden zitiert: Schmierer, Arbeiterbildung; Cbristof Rieber Das Sozialistengesetz und die Sozialdemokratie in Württemberg 1878-1890 (Schriften zur südwestdeutschen Landeskunde, Bd. 19, Stuttgart 1984), im folgenden zitiert: Rieber, Sozialistengesetz; Maja Christ-Gmelin, Die württembergische Sozialdemokratie 1890-1914. Ein Beitrag zur Geschichte des Reformismus und Revisionismus in der deutschen Sozialdemokratie (Diss. phil. Stuttgart 1976). Die drei Autoren haben ihren Gegenstand auch in dem Sammelband „Die SPD in Baden-Württemberg und ihre Geschichte. Von den Anfāngen der Arbeiterbewegung bis heute“, Hrsg. Jörg Scbadt und Wolfgang Schmierer (Stuttgart 1979) behandelt.
} 
seine Eigenheiten, seine historische Herkunft preisgegeben. Die Herausbildung einer Arbeiterpartei von eigener Statur und Organisation ist ein Kapitel dieser Geschichte, welche von Beharrung und Widerstand berichtet und doch mit der Anpassung an die vorherrschenden Verhältnisse ihr Ende fand.

Schon 1863, als ADAV und Vereinstag die Arbeiterbewegung auf eine neue, eine institutionelle Grundlage stellten, als aus der Konkurrenz beider Gründungen jenes Spannungsfeld erwuchs, das für sechs Jahre die Entwicklung bestimmte - schon damals galt in Württemberg der Primat der politischen Partei, und das hieß: der bürgerlich-liberalen Partei. Die Arbeitervereine, so die Abgrenzung gegen die Lassalleschen Bestrebungen, hătten mit parteimäßiger Organisation nichts zu schaffen. Ihre Zwecke seien gewerbliche Bildung, genossenschaftliche Selbsthilfe sowie die Bekundung sozialer Interessen; ihr politisches Credo sei das allgemeine Wahlrecht. Der Arbeiter sei Gefolgsmann der Fortschrittspartei: so forderte August Hochberger, Sprecher seines Standes in jenen frühen Jahren ${ }^{2}$.

Auch die Spaltung des Fortschritts, wie sie durch die Bismarcksche Politik der mittleren sechziger Jahre hervorgerufen wurde, hat die Rollenverteilung nicht eigentlich gefährdet. Wohl aber hat sie die allgemeine Politik in die Arbeitervereine hineingetragen. Wo zwei Parteien sich um deren Loyalitāt bemühten, war das Politische von ihnen nicht mehr fernzuhalten. Andererseits wurde das Prinzip der Gefolgschaft durch die Konkurrenz politischer Gruppen nicht in Frage gestellt oder gar gefährdet. Seit 1866 begannen sich die parteipolitischen Präferenzen zu spalten, wobei örtliche Gegebenheiten und persönliche Konnexionen den Entscheidungen nicht selten die Richtung gaben. Als im Juli 1868 ein Landtag gewählt wurde - der erste in der Geschichte des Landes nach dem allgemeinen Stimmrecht -, votierten die Vereine von Stuttgart und Cannstatt für die „Preußen“, die von Reutlingen, Rottweil und Hall für die Volkspartei ${ }^{3}$.

Nach den Gründerjahren 1863/64, nach den Jahren politischer Spaltung 1865-68 sodann folgte eine letzte Phase des Jahrzehnts, in welcher die Arbeitervereine fast gänzlich von der Volkspartei ins Geschirr genommen wurden. Es ist jene Zeit, da diese Partei vom populären Anti-Preußen-Affekt zehrt, da sie durch Organisation, publizistischen Gestus und durch Kampagnen den Typus Partei in seiner avanciertesten Form repräsentiert.

Die württembergische Volkspartei war Teil jenes bismarckkritischen Reflexes auf der Linken, der sich allenthalben in jenen Jahren in Deutschland hervortat, der in lok-

2 Schmierer, Arbeiterbildung, 61.

${ }^{3}$ Ebd., $114 \mathrm{f}$.; Gerlinde Runge, Die Volkspartei in Württemberg von 1864-1871. Die Erben der 48er Revolution im Kampf gegen die preußisch-kleindeutsche Lösung der nationalen Frage (Veröffentlichungen der Kommission für geschichtliche Landeskunde in Baden-Württemberg. Reihe B, Forschungen, Bd. 62, Stuttgart 1970) $153 \mathrm{ff}$, im folgenden zitiert: Runge, Volkspartei; Dieter Langewiesche, Liberalismus und Demokratie in Württemberg zwischen Revolution und Reichsgründung (Beiträge zur Geschichte des Parlamentarismus und der politischen Parteien, Bd. 52, Düsseldorf 1974) 376, im folgenden zitiert: Langewiescbe, Liberalismus; Hartwig Brandt, Parlamentarismus in Württemberg 1819-1870. Anatomie eines deutschen Landtags (Handbuch der Geschichte des deutschen Parlamentarismus, hrsg. v. Gerbard A. Ritter, Düsseldorf 1987) 175, im folgenden zitiert: Brandt, Parlamentarismus. 
keren Parteigründungen (Demokratische Volkspartei 1863 66; Süddeutsche Volkspartei 1868 ff.) institutionelle Form erhielt, der aber nur in Württemberg und Sachsen zu einer politischen Kraft im Staate gedieh ${ }^{4}$. Freilich tritt in beiden Parteien nun auf antipodische Weise das Verhältnis von liberalen und interventionistischen, von besitzschützenden und besitzverteilenden Bestrebungen hervor. Auch die sãchsische Volkspartei hatte 1866 zuvörderst allgemeines Wahlrecht und Volkswehr, die klassischen Eingebungen der Demokratie, in ihr Programm geschrieben und die sozialen, die sozialverändernden Ziele hintangestellt. Aber die Partei, schon damals unter der Führung Bebels und Liebknechts stehend, war doch aus Arbeitervereinen hervorgegangen und binnen kurzem auch organisatorisch mit diesen verflochten, wohingegen die württembergische Rivalin sich in jedem Belang als Exponentin des Besitzbürgertums zeigte, weiche der Arbeiterschaft eher aus taktischen Erwägungen Avancen machte. Die Ziele der Partei, so Karl Mayer, ihr erster Stratege und Agitator, seien Abbau der indirekten Steuern und Abschaffung des stehenden Heeres ${ }^{5}$.

Wiewohl der Nümberger Vereinstag vom September 1868, eine erste Demonstration der sāchsischen Richtung, im Grunde bereits die Fronten geschieden hatte, zeigte die Volkspartei weder Bedürfnis noch Interesse, einer möglichen Abspaltung durch programmatisches Entgegenkommen vorzubeugen. Freilich betrachteten sich die württembergischen Arbeitervereine zu jener Zeit selbst - wenn sie auch ihre eigene Organisation aufrechterhielten - als Flügel der Partei. Es bedurfte also nicht eigener Anstrengungen der bürgerlichen Meinungsführer, ihre Klientel vor „Nürnberger“ oder spāter „Eisenacher“ Anfechtungen zu bewahren.

Die Eisenacher Parteigründung vom August 1869, deren Impulse aus der sächsischen Volkspartei kamen, welche ihrerseits dann in der Nachfolgerin aufging, trieb die württembergische Volkspartei in die nationale Isolation. Organisatorisch, weil der zentralistische Zugriff der SDAP die föderalistische Struktur des Verbandes deutscher Arbeitervereine beseitigte und die Inkorporation der württembergischen Volkspartei, die sich ja selbst auf dem Wege zur modernen Mitgliederpartei befand, dieser ihren Charakter zu nehmen drohte; programmatisch, weil die forcierte Beschränkung auf die Vertretung eines Arbeiter-Klasseninteresses in Württemberg - schon der vielfältigen sozialen Überschneidungen halber - eher Unverständnis hervorrief, jedenfalls, aufs Ganze gesehen, der nötigen Stützung entbehrte.

So war die Trennung nicht mehr aufzuhalten ${ }^{6}$. Und als sie unvermeidbar geworden, hat der „Beobachter“, das Parteiorgan, ihr nach Kräften nachgeholfen. Karl Mayer

4 Rolf Weber, Kleinbürgerliche Demokraten in der deutschen Einheitsbewegung 1863-1866 (Berlin 1962) 48 ff.; ders., Demokratische Volkspartei 1863-1866, in: Dieter Fricke u. a. (Hrsg.), Lexikon zur Parteiengeschichte, Bd. 1 (Leipzig 1983) 504-13; Ludwig Elm, Süd-Deutsche Volkspartei (SDVp), in: Bd. 4 (1986) 171 ff.; Dieter Fricke, Verband Deutscher Arbeitervereine (VDAV) 1863-1869, in: ebd., Bd. 4 (1986) 253-65

${ }^{5}$ Zu K. M.: Runge, Volkspartei, 27 ff. u. ö.; Langeuiesche, Liberalismus, 91 f. u. ö.; Brandt, Parlamentarismus, 715-22.

${ }^{6}$ Die Stationen dieser Spaltung beschreibt der - lāngst zum Klassiker gediehene - Aufsatz von Gustav Mayer: Die Trennung der proletarischen von der bürgerlichen Demokratie in Deutschland 1863-1870, in: Archiv für die Geschichte des Sozialismus und der Arbeiterbewegung, Bd. 2 (1912) 1-67 (Neuabdr. in: Gustav Mayer, Sozialismus und bürgerliche Demokratie, hrsg. v. Hans- 
schrieb, jenes berühmte Diktum der Internationalen Arbeiter-Assoziation nutzend, das die Abschaffung des Privateigentums an Grund und Boden gefordert hatte und das die SDAP unwidersprochen ließ, eine flammende Artikelfolge wider die Basler Beschlüsse?

Die Volkspartei erlitt nach der Trennung von Eisenach Verluste, aber sie blieb als Repräsentantin der Arbeiterschaft in der Vorhand. Nur acht Arbeitervereine traten bis zur Jahresmitte 1870 der Eisenacher Neugründung bei (Stuttgart, Metzingen, Cannstatt, Eßlingen, Giengen, Hall, Ravensburg, Tettnang). In mehr als der Hälfte verfügte die Volkspartei weiterhin über die Mehrheit der Stimmen ${ }^{8}$.

Ob diese indes auch auf weitere Sicht ihre Position hätte behaupten können, steht dahin und bleibt ein Thema für Spekulationen. Halten wir uns an das Geschehen selbst, so hat der deutsch-französische Krieg die Konkurrenz ex abrupto beendet und den Eisenachern den Weg nach Württemberg freigemacht. Angesteckt von der Kriegshysterie, war die Volkspartei noch in letzter Stunde ins preußisch-nationalistische Lager gewechselt und hatte dadurch jene Befindlichkeit erst provoziert, die Wilhelm Liebknecht ihr schon zuvor prophezeit hatte: daß sie zwischen den Mühlsteinen Sozialdemokratie und Nationalliberalismus zerrieben werde?

Moralisch und organisatorisch ruiniert, ging die Partei aus dem Krieg hervor. Die Zahl ihrer Vertreter im Reichstag schwankte in den siebziger Jahren zwischen einem und drei, ganze sieben Mandate errang sie 1876 bei den Landtagswahlen. Die Landesversammlung, der klassische Anzeiger innerparteilicher Vitalität, kam über Jahre hinweg zum Erliegen ${ }^{10}$.

Wie in den sechziger Jahren der deutsche Liberalismus allgemein so hat in den siebziger Jahren die württembergische Volkspartei ein Vakuum hinterlassen, in welches eine Arbeiterpartei sensu stricto hineinzustoßen und sich als Organisation zu etablieren vermochte. Freilich geschah dies nun aus kleinsten Anfãngen und vielfach fast vom Nullpunkt aus. Während Stuttgart von den Lassalleanern erobert wurde, faßten die Eisenacher im Neckartal Fuß: in Eßlingen zunächst, in Göppingen und im Remstal danach. Abseits der Verkehrs- und Industrieschiene Heilbronn-Reutlingen hat die Sozialdemokratie gleichwohl über Jahrzehnte kaum an Boden zu gewinnen vermocht.

Ulrich Webler, Frankfurt 1969, 108 ff.), im folgenden zitiert: Mayer, Trennung. Zur neueren (und vergleichenden) Diskussion: Wolfgang Schieder, Das Scheitem des bürgerlichen Radikalismus und die sozialistische Parteibildung in Deutschland, in: Hans Mommsen (Hrsg.), Sozialdemokratie zwischen Klassenbewegung und Volkspartei (Frankfurt/M. 1974) 17-34.

${ }^{7}$ Beobachter, Nr. 238-40 v. 13./14./15.10.1869. S. a. Schmierer, Arbeiterbildung, 144.

${ }^{8}$ Ebd., 150 f.

${ }^{9}$ Mayer, Trennung, $60 \mathrm{f}$.

${ }^{10}$ Eine Biographie der Volkspartei zwischen 1871 und 1890 fehlt. Die Rungesche Arbeit schließt mit der Reichsgründung, die Studien von Klaus Simon (Die württembergischen Demokraten. Ihre Stellung und Arbeit im Parteien- und Verfassungssystem in Württemberg und im Deutschen Reich 1890-1920, Veröffentlichungen der Kommission für geschichtliche Landeskunde in Baden-Württemberg, Reihe B, Forschungen, Bd. 52, Stuttgart 1969) im folgenden zitiert: Simon, Demokraten, und James Clark Hunt (The People's Party in Württemberg and Southern Germany 1890-1914, Stuttgarter Beiträge zur Geschichte und Politik, Bd. 9, Stuttgart 1975) im folgenden zitiert: Hunt, People's Party, beginnen zwanzig Jahre danach. 
Institutionelles Gehāuse ihrer Bestrebungen waren die herkömmlichen Arbeitervereine, aber auch diese fielen der Partei nicht in dem Maße zu, wie sie des Einflusses der Volkspartei entrieten. Sie wurden in der Mehrzahl unpolitisch, oder sie pflegten die alte, die liberale Sozialpolitik Schulze-Delitzscher, Pfeifferscher Provenienz. In den ersten Jahren - ca. 500 Mitglieder hat Wolfgang Schmierer für 1872 gezählt $^{11}$ - sind SDAP und ADAV kaum über die Stützpunkte des Reichsgründungsjahres hinausgelangt. Verglichen mit den Verhältnissen anderer Regionen, von Hamburg und Sachsen nicht zu reden, war der Zuwachs an Mitgliedern und Wahlstimmen gering. Erst 1895 gewann die Partei ihren ersten Sitz im Landtag, erst 1898 gar ein Mandat im Reichstag. Weder bei den Wahlen dort noch bei den Wahlen hier ging der Anteil an Stimmen über $4 \%$ hinaus $^{12}$.

Die Gründe für diese schleppende Entwicklung sind verschiedener Art. Da war der Anteil von Beschäftigten in der Landwirtschaft, der jenem in der Industrie mit 45,1 zu $35 \%$ voraufging und gegenüber der Reichsziffer ( $35 \mathrm{zu} 38,5 \%$ ) eine Besonderheit darstellte $^{13}$. Da war zum zweiten der Anteil der ökonomisch Selbständigen, welcher in Württemberg 1895 mit 37,4\% immer noch um neun Punkte über dem Anteil im Reiche $\operatorname{lag}^{14}$. Da war zum dritten, und von dem ersten und zweiten nicht zu trennen, die starke Position Selbständiger in der Repräsentanz der Partei, wohingegen unter den Mitgliedern von Anbeginn der Handwerker-Arbeiter überwog. (Bronnenmeyer, nennen wir ihn beim Namen, war der erste Vertreter dieser Schicht, der sich - 1884 - um ein Reichstagsmandat bemühte). Da war viertens der hohe Anteil von Nicht-Württembergern unter Mandatsträgern und Wahlbewerbern. (Erst 1877 ist mit dem Eßlinger Julius Motteler ein Einheimischer auf den Kandidatenlisten zu finden.) Und da war fünftens und schließlich der eher bedächtige Stil der Auseinandersetzung, das Fehlen aller theoretischen Ambition. Lassalle hat niemals große Resonanz erzeugt. Die Bebelsche Richtung, freilich wohl eher Bebel selbst, der häufig in Württemberg weilte, war geschätzt. Aber in der Praxis war die Tuchfühlung zur Volkspartei ein Korrektiv, das alle ideologischen Festlegungen relativierte. Daß Albert Dulk, Königsberger, Schriftsteller und Freidenker, dreimal als Parlamentsbewerber auf den Schild gehoben wurde, dies erscheint durchaus als eine plausible Quintessenz des Gesagten.

Bei alledem war die württembergische Sozialdemokratie indes keine Quantité négligeable im Parteiensystem der siebziger und achtziger Jahre. Die zählbaren Erfolge waren gering, aber das Mehrheitswahlrecht in Reich und Land war einer vom Nullpunkt

\footnotetext{
11 Schmierer, Arbeiterbildung, 180.

12 Die Geschichte der Reichstags- und Landtagswahlen (seit 1871) in Württemberg bedarf noch der Erforschung. Die vorliegende Skizze stützt sich in ihren Zahlenangaben auf Wolfgang Schulte, Struktur und Entwicklung des Parteisystems im Königreich Württemberg (Diss. phil. Mannheim 1970), im folgenden zitiert: Scbulte, Parteisystem; Gerbard A. Ritter unter Mitarbeit von Merith Niebuss, Wahlgeschichtliches Arbeitsbuch. Materialien zur Statistik des Kaiserreichs 1871-1918 (München 1980), im folgenden zitiert: Ritter, Arbeitsbuch und die genannten Arbeiten von Scbmierer und Rieber.

${ }^{13}$ Rieber, Sozialistengesetz, 39.

14 Gerd Hoborst/Jürgen Kocka/Gerbard A. Ritter, Sozialgeschichtiches Arbeitsbuch. Materialien zur Statistik des Kaiserreichs 1870-1914 (München 1975) 70 f.
} 
emporstrebenden Partei eben auch nicht günstig ${ }^{15}$. 3,8, 2,4, 2,8, 3,6, 3,5\% - so lauten die Anteile bei den Reichstagswahlen von 1874 bis $1887.1,3,1,4,4,3$, heißen sie bei den Landtagswahlen des gleichen Zeitraums. Aber diese Zahlen verhüllen den Abstand, der die industriellen Zentren des Neckartals von den arbeiterfernen Regionen Hohenlohes, Oberschwabens, des Schwarzwaldes - trennte. In Stuttgart gewann der ADAV bei den Reichstagswahlen 1874 16,7\%, die SDAP in Göppingen 20\%, in Eßlingen gar 27,3\% der Stimmen. Ein Jahr später - bei einer Nachwahl in Stuttgart - erhöhte die vereinte Partei ihren Anteil auf 26,3 v.H. Uber dieses Niveau ist sie dann erst 1893, nun allerdings in kräftigen Schüben - 20,3, 27,4, 27,9, 32,5\% - hinausgelangt, wobei es ein Charakteristikum bleibt, daß die Zuwachsraten der Landtagswahlen denen der Reichstagswahlen immer erst im zeitlichen Abstand einiger Jahre folgten.

Indessen ist die Rolle des Nachzüglers, welche die württembergische Sozialdemokratie im Troß der Bewegung über Jahrzehnte spielte, nur ein Teil dessen, was ihre Besonderheit ausmachte. Das andere ist die eigentümliche Verschränkung von Miteinander und Streit, die ihr Verhältnis zur benachbarten Volkspartei ausmachte. Keine Frage ist, daß sie von der Depression dieser Partei in den siebziger Jahren profitierte und bürgerliche Protestwähler an sich zog, die den Bismarckkult der Deutschen Partei nicht schätzten. Keine Frage aber auch, daß die Politik der Wahlempfehlungen hüben und drüben, die Koalitionsfähigkeit also von dort nach hier und von hier nach dort das Parteiensystem elastisch hielten, ja ein Klima der Gemeinbürgerlichkeit konservierten, welches im Lande eine Tradition, aber im System des Kaiserreichs eigentlich keine Bleibe hatte.

Die ersten Absprachen, die eine Phase des Profits der SDAP an der Malaise der Volkspartei - so 1874 in Stuttgart - beendeten, datieren von 1876. Bei der Landtagswahl dieses Jahres verzichtete die SDAP in Eßlingen auf einen Kandidaten zugunsten der Volkspartei, deren Bewerber - Karl Mayer - gewann. In Stuttgart geschah es umgekehrt, wo Albert Dulk indessen das Mandat verfehlte. Immerhin war erstmals ein sozialdemokratischer Bewerber in die Stichwahl gelangt, Kandidat von Linksbürgerlichen und Arbeiterpartei zugleich.

Auch bei der Reichstagswahl des folgenden Jahres gab es Vereinbarungen, wiewohl eine Debatte in der SAP erstmals auch die fragwürdigen Seiten solcher Unterstützungen offenbarte. In Reutlingen hatte die Partei dem aufstrebenden Volksparteiler Friedrich Payer zum Mandat verholfen. In Eßlingen war Friedrich Retter, ein Posthalter aus Ellwangen, der Begünstigte. An beiden Hilfestellungen, da sie ohne Gegenleistung blieben, entzündete sich Kritik in der Partei.

In den 80er Jahren bestimmten solche Ungleichgewichte noch stärker das Bild. Zweimal (1881 und 1883) verschaffte sozialdemokratische Unterstützung - und damit der eigene Verzicht - dem Demokraten Sigmund Schott das Stuttgarter Mandat, wohingegen die Volkspartei (Heilbronn 1884, Cannstatt 1887), wo sie selbst auf die Konkurrenz verzichtete, ihren Anhängern Wahlenthaltung nahelegte. Wo das Sozialistengesetz der SAP alle Versammlungstätigkeit untersagte und Handhaben für Preßunter-

15 Zahlen nach Schmierer, Arbeiterbildung, 274f. (Reichstagswahlen); Ritter, Arbeitsbuch, 89 (Reichstagswahlen); Rieber, Sozialistengesetz, 799-805 (Reichstags- und Landtagswahlen); Hunt, People's Party, 180-82 (dsgl.). Auf Einzelbelege wird im folgenden verzichtet. 
drückung und Ausweisung bot, da zehrte diese von der Ablehnung des Gesetzes über die Parteigrenzen hinweg. Daß Redner der SAP auf Wahlversammlungen der Volkspartei auftreten konnten - und aufgetreten sind, war in Grenzen ein Ausgleich für die Stimmenunterstützung, welche den Demokraten zugute kam. Die achtziger Jahre boten, das Verhăltnis beider Parteien zueinander betreffend, eine Umkehrung von Gewinn und Verlust. War die SDAP in den frühen siebzigern Begünstigte der politischen Indolenz der Demokraten, so zogen diese Profit aus der Kriminalisierung sozialdemokratischer Politik ein Jahrzehnt danach. Die nur wenig differierenden Stimmengewinne von SDAP und SAP zwischen 1870 und 1890 geben eine Stagnation des Wählerverhaltens vor, die es vermutlich nicht gab. Hier hatten abtrünnige Teile des Bürgertums den Stimmenfundus besorgt, dort der durch Verfolgung geschärfte proletarische Gemeinsinn. Dazu war es die fortschreitende Industrialisierung selbst, welche den Sozialdemokraten mit Stetigkeit neue Hilfstruppen zuführte.

Das politische System Württembergs bestand in den 70/80er Jahren aus einem Regierungschef - Mittnacht - , der sich vom Landtag und vom Landesherrn fast emanzipiert hatte, gleichwohl im Parlament über eine Anhängerschaft von Gouvemementalen und großdeutschen Konservativen verfügte. Es bestand zum zweiten aus einer nationalen Partei, die sich in den Jahren der Reichsgründung als deren Streiter und Agent verbraucht hatte und sich zunehmend - als Hölder 1881 in das Innenministerium eintrat, wurde es offenbar - zur Regierungspartei wandelte. Und es verfügte drittens über eine demokratische Opposition, die, parlamentarisch indes eher schwach, auf der Linken in einer Arbeiterpartei neuen Typs, der SAP also, eine Fortsetzung fand.

Dieses System hat sich nach 1890 von Grund auf verändert. Zum einen gab es eine Renaissance der Volkspartei, welche, die Reichsernüchterung nutzend, zur stärksten politischen Kraft des Landes wurde und im Landtag über ein Drittel, im Reichstag fast ein Jahrzehnt lang über mehr als die Hālfte der württembergischen Mandate verfügte. Da war zum zweiten der politische Katholizismus, der bis dahin - Folge der schon in den sechziger Jahren entschärften Kirchenfrage - in getrennten Lagern agiert hatte und sich erst 1895, großdeutsche Konservative und großdeutsche Liberale vereinigend, als Landes-Zentrumspartei organisierte. Da waren drittens die Geschlagenen des rechten Lagers, die geschrumpfte Deutsche, nun nationalliberal getaufte Partei, Reste der gouvernementalen Landespartei, Agrarkonservative, Ritterschaft, evangelische Prālaten, die sich zur sog. „Freien Vereinigung“ zusammenschlossen ${ }^{16}$. Und da war viertens die SPD, deren Stimmenanteile bei den Reichstagswahlen nach 20 und $27 \%$ 1912 mit 32,5 v. H. ihren Zenit erreichten.

Das Verhältnis der beiden Linksparteien, zuvor ein solches der Minderheiten, der Verlierer, hatte sich gewandelt. Mit gewachsener Stimmenstärke trat indessen nun

16 Simon, Demokraten, $47 \mathrm{ff}$.; Hunt, People's Party, $71 \mathrm{ff}$.; David Blackbourn, Class, Religion and Local Politics in Wilhelmine Germany. The Centre Party in Württemberg before 1914 (Veröffentlichungen des Instituts für Europäische Geschichte Mainz, Abt. Universalgeschichte, BH 9, Wiesbaden 1980); Georg H. Kleine, Der württembergische Ministerpräsident Frhr. Hermann von Mittnacht, 1825-1909 (Veröffentlichungen der Kommission für geschichtliche Landeskunde in Baden-Württemberg, Reihe B, Forschungen, Bd. 50, Stuttgart 1969) $30 \mathrm{ff.}$ 
auch das Geschäftliche, das taktische Kalkül ihrer Beziehungen schärfer hervor. Bei den Reichstagswahlen von 1890 gewann die Volkspartei zwei der 17 zu vergebenden Mandate im ersten Durchgang, 7 weitere im zweiten gegen konservative Kandidaten, obwohl diese in 5 Fällen zunächst in Front gelegen hatten. Wenigstens drei, vermutlich vier der zugewonnenen Sitze verdankte sie sozialdemokratischer Unterstützung. Ähnlich waren die Verhältnisse 1893. Umgekehrt hatte die SPD in Stuttgart mit ihrem Kandidaten in beiden Fällen das Nachsehen, obwohl dieser als stärkster Bewerber aus der ersten Wahl hervorgegangen war, in der Stichwahl dann aber dem Mann der Liberalen unterlag. Nicht mehr als $40 \%$ der VP-Wählerschaft, so hat man vermutet ${ }^{17}$, dürfte sich auf die Wahlempfehlung ihres Ortsvereins eingelassen haben. 1898 gewann die SPD Stuttgart im ersten Anlauf, unterlag aber in drei Stichwahlen, zu denen sie vorgedrungen war, gegen den konservativen Kandidaten. Umgekehrt war sie in vier Wahlkreisen jene Kraft, welche für den Sieg der Volkspartei am Ende den Ausschlag gab ${ }^{18}$.

Was die Beispiele zeigen, ist eine einseitige, eine schiefe Form der Kooperation, die freilich darin ihre Ursache hatte, daß die Volkspartei - ihren Zielsetzungen wie ihrer Wählerschaft nach - sich in der Mitte zwischen zwei Parteien befand. Wo es um die Entscheidung zwischen Volkspartei und Nationalliberalen ging, votierte die SPD stets für die erstere. Standen die Wähler der Volkspartei vor der Entscheidung, für links oder rechts zu votieren, so taten sie es mit "wechselnder Gewichtung “19. Das empfehlende Votum der Gremien wog in solchen Fällen gering.

Freilich ist dies nicht die ganze Erkenntnis, welche der Gegenstand bereithālt. In einer tieferen Schicht beginnt zur gleichen Zeit jener Prozeß, dessen Auswirkungen indessen erst nach der Jahrhundertwende in Gänze zutage treten und den man als die Milieuverfestigung der Parteien bezeichnen kann - und bezeichnet hat ${ }^{20}$. Die württembergische Parteiengeschichte der 1890er Jahre zeigt einen Höhenflug der föderalen Demokratie und einen fortschreitenden Stimmenzuwachs ihres Partners und Kontrahenten, der SPD. War das Wahlverhalten der württembergischen Wählerschaft bis in die sechziger Jahre von sozialständischen Fixierungen noch weitgehend frei und waren die Parteien eher durch Verfassungssätze und nationale Präferenzen geschieden, so hat sich diese Tradition auch in den siebziger und achtziger Jahren z.T. noch fortgesetzt, wiewohl der Zusammenhang von Industrialisierung und sozialdemokratischer Stimmenmacht diesem System schon den Boden zu entziehen begann. Beeindruckt von diesem Umstand und der Perspektive, die er zu eröffnen schien, zudem einer sozialpolitischen Gegenoffensive nicht mehr fähig, suchte die Volkspartei nach 1900 eine soziale Kompensation, und sie fand sie, wo auch eine Bauernpartei seit 1898 kandidierte und das Zentrum längst sein Feld abgesteckt hatte, im Mittelstand. Seitdem ist die soziale Verfestigung auch in Württemberg eine Konstante der Parteiengeschichte.

${ }^{17}$ Hunt, People's Party, 121.

${ }^{18}$ Ebd.; Schulte, Parteisystem, 134 ff.; Rieber, Sozialistengesetz, 689 ff.

19 Schulte, Parteisystem, 136.

${ }^{20}$ M. Rainer Lepsius, Parteisystem und Sozialstruktur: zum Problem der Demokratisierung der deutschen Gesellschaft (1966, Wiederabdr. in: Gerbard A. Ritter (Hrsg.), Die deutschen Parteien vor 1918, Köln 1973, 56-80). 